\title{
Que reste t'il du côlon tropical en 2013 ?
}

\author{
F. Klotz $\cdot$ P.S. Mbaye $\cdot$ C. Klotz \\ (C) Springer-Verlag France 2013
}

Les médecins qui participèrent à l'expansion des empires coloniaux sous les tropiques, décrivirent de manière imagée et souvent morbide cette pathologie colique qui atteignait les autochtones et décimait les rangs des étrangers.

Dutrouleau en 1861 dans son « traité des maladies des européens dans les pays chauds », montrait avec force détails les divers aspects de la pathologie intestinale dont « la dysenterie de Cochinchine » qui n'était autre que l'amibiase intestinale..

De nombreux médecins coloniaux firent progresser l'épidémiologie, les descriptions cliniques et les traitements de ces maladies coliques tropicales et de leurs séquelles dont l'origine d'abord mystérieuse : « les miasmes du sol tropical » fut progressivement rattachée à des parasites : l'amibe, le schistosome, les némathelminthes, ou à des bactéries cosmopolites : la shigelle, le bacille de Koch.

Cette pathologie intestinale paraissait amplifiée dans sa fréquence et sa sévérité par le climat, la pauvreté et ses attributs : la promiscuité, le manque d'hygiène, l'analphabétisme.

Jusqu'aux « années 70 », les thérapeutiques étaient soit peu efficaces, soit sources d'effets secondaires importants (anthiomaline, émetine, niridazole), avec comme corollaire, une mauvaise observance, une diffusion des infections et un grand nombre d'états pathologiques chroniques.

L'amibiase intestinale, si bien décrite dans le traité de Blanc et Siguier en 1950, est restée dans sa forme chronique et dans la colopathie post amibienne séquellaire, un véritable stigmate des fonctionnaires civils et militaires et des « aventuriers » qui avaient vécu aux colonies. Cette pathologie couta cher à l'état français en pensions d'invalidité et fit les beaux jours des stations thermales.

F. Klotz $(\bowtie)$

Professeur au Val de Grâce, Paris

e-mail : fklotz2008@yahoo.fr

P.S. Mbaye

Professeur agrégé du Val de Grâce, Chef des Services médicaux, hôpital Principal de Dakar, Sénégal

C. Klotz

Interne des hôpitaux des Armées HIA du Val de Grâce, Paris
Depuis plusieurs décennies l'apparition de molécules très efficaces et peu toxiques permettent de traiter très facilement toutes ces affections : le métronidazole pour l'amibiase, le praziquantel pour la bilharziose, les benzimidazolés pour les helminthiases, les antibiotiques spécifiques ou non pour la tuberculose et la shigellose. Mais malgré ces traitements, malgré les moyens endoscopiques, biologiques, anatomopathologiques, rendant le diagnostic aisé et sûr, la pathologie infectieuse colique des populations autochtones sous les tropiques s'est perennisée avec l'aggravation de la pauvreté et de l'urbanisation anarchique, avec les grands travaux d'irrigation et l'instabilité sociopolitique. Elle s'est enrichie de la pathologie liée au sida, dont le berceau est tropical. Le côlon est une des cibles privilégiées du virus de l'immunodéficience humaine et de ses affections opportunistes satellites : parasitaires (cryptosporidies), virales (cytomégalovirus), bactériennes (mycobactéries atypiques) et tumorales (sarcome de Kaposi et lymphomes). Toutes ces affections dont les thérapeutiques, si toutefois elles existent, sont difficilement accessibles pour les pays en voie de développement.

Les moyens modernes d'investigation ont permis de mettre en évidence ou de saisir l'émergence de pathologies longtemps méconnues sous les tropiques, les maladies chroniques inflammatoires intestinales (MICI) avec là encore des impasses thérapeutiques par défaut de moyens.

Le côlon tropical a changé de visage pour l'occidental « missionnaire » ou voyageur ; il n'est plus un mal redouté. Pour les autochtones souvent atteints par les nouveaux fléaux de l'immunodépression et souvent totalement démunis d'armes thérapeutiques, il s'est enrichi dans son polymorphisme et aggravé dans sa morbidité.

Le $\mathrm{XXI}^{\mathrm{e}}$ siècle verra t'il l'hygiène fécale s'installer sous les tropiques, un vaccin contre le sida et contre la bilharziose être distribué dans les pays en voie de développement? Permettons-nous de rêver et de douter! 